\title{
Inhibitory effects of instrument-assisted neuromobilization on hyperactive gastrocnemius in a hemiparetic stroke patient
}

\author{
Jeong Jae Lee, Jae Jin Lee, Do Hyun Kim and Sung (Joshua) Hyun You* \\ Department of Physical Therapy, Yonsei University, Wonju City, Kangwon-do, \\ Republic of South Korea
}

\begin{abstract}
An instrument-assisted soft tissue mobilization (IASTM) technique has recently been used specifically to inhibit hypertonic muscles and to lengthen muscle fiber shortness. However, it is unknown whether IASTM will show such promising inhibition effects on excessive ankle plantarflexion following stroke. Therefore, the purpose of this study was to use electromyographic (EMG) analysis to determine the ability of IASTM to reduce gastrocnemius (GCM) hypertonicity and concurrently facilitate tibialis anterior (TA) lengthening in a stroke patient. EMG activity on the patient's TA and GCM was measured before and after applying IASTM. After the intervention, the GCM was deactivated by 43\%, and TA activity increased by $150 \%$, indicating IASTM-induced inhibition of the overactive GCM. The neuromobilization technique using IASTM showed a promising improvement of neuromuscular imbalance between TA and GCM activations, which can increase gait performance in a stroke case.
\end{abstract}

Keywords: Instrument-Assisted Soft Tissue Mobilization (IASTM), Electromyography (EMG), stroke, gait

\section{Introduction}

Excessive ankle plantarflexion is an important hallmark locomotion impairment often associated with a high risk of fatal falls in spastic stroke [1-3]. Cortical dis-inhibition occurs after cerebral diaschisis and decreases inhibitory control over the excitatory interneurons in the spinal cord, which transmits excitatory neuronal signals via alpha and gamma motor neurons innervating plantarflexors. Subsequently, the excitatory neuronal impulses contribute to hypertonicity or overactivation of the antagonist (e.g., gastrocnemius, GCM) and resulting in reciprocal inhibition of the agonist (e.g., tibialis anterior, TA), thereby leading to excessive ankle plantarflexion [4-7]. Over time, this excessive ankle plantarflexion can result in soft tissue (muscle, tendon, ligaments, fascia, and skin) shortness and contraction during locomotion [8-10]. Neurophysiological mechanisms underlying hypertonicity or over-activation of the antagonist post-stroke have not been fully elucidated, but prior work suggests that the reduction of the hyper-activated antagonist GCM might help mitigate excessive

\footnotetext{
*Corresponding author: Joshua (Sung) H. You, Department of Physical Therapy, Yonsei University, Wonju City, Kangwon-do 220-710, Republic of South Korea. Tel.: 033-760-2476; Fax: 033-760-2496; E-mail: joshuayou7@gmail.com.
}

0959-2989/14/\$27.50 @ 2014 - IOS Press and the authors. 
plantarflexion and concurrently facilitate the underactive TA, which will improve foot clearance during the swing phase of the gait cycle [3,11-14].

Neuromobilization techniques, including hold-relax (HR), strain-counterstrain (SCS), and soft tissue mobilization (STM), have been commonly used to improve hypertonicity and associated flexibility [15-17], but the results were variable. Moreover, the conventional manual neuromobilization techniques are often physically demanding, which can result in tenosynovitis or other musculoskeletal impairments among clinicians if improper biomechanics is repetitively used over time [18].

To mitigate the musculoskeletal risks, an instrument-assisted soft tissue mobilization (IASTM) technique has recently been used because it provides superior merits to specifically inhibit the hypertonic muscle and lengthen muscle fiber shortness [19]. An electromyographic (EMG) study showed decreased excessive muscle activation and improved knee terminal extension movement and strength after application of IASTM in subjects with hamstring shortness [20]. However, it is unknown whether IASTM would produce such promising inhibition effects in excessive ankle plantarflexion following stroke. Therefore, the purpose of this study was to use EMG analysis to determine the effects of IASTM on GCM hypertonicity and TA facilitation in a stroke patient.

\section{Methods}

A 22-year-old male was admitted to a university hospital for evaluation of acute ischemic stroke in 2011. He presented with acute onset numbness and tingling of right upper and lower extremities and mild expressive aphasia. Magnetic resonance imaging (MRI) of the brain demonstrated a left-sided acute hemorrhage in the middle cerebral artery territory, which involved moderate lesions along the corticospinal tract (corona radiata, posterior limb of the internal capsule) (Figure 1). Currently, clinical neurological tests revealed muscle weakness on the right upper and lower extremities (e.g., triceps, TA) and hypertonicity on biceps and GCM muscles (Modified Ashworth Scale: 1+ to 2), which affected locomotion, primarily due to neuromuscular imbalance between the overactive GCM and underactive TA. His sensation, vision, cognitive, and mental functions were relatively intact. Ethical approval for the study was granted by the Yonsei University Wonju Campus Human Studies Committee.

\section{Procedure}

Surface EMG data were collected using a WEMG-8-type (LAXTHA Inc., Daejeon, Korea). Before electrode attachment, skin was prepared by shaving, cleaning with an alcohol prep pad, and sanding. A pair of active electrodes was attached on the muscle zone in parallel, and a reference electrode was attached on the patella. A pair of active electrodes with a diameter of $1.8 \mathrm{~cm}$ and an inter-electrode distance of $2 \mathrm{~cm}$ was placed on the two muscles on the right leg: the TA and GCM. The TA electrodes were placed on the anterior, lateral, and superior aspects of the tibia at approximately one-third the distance between the knee and ankle. Two active electrodes were placed parallel and just lateral to the medial shaft of the tibia, at one-quarter to one-third the distance between the knee and ankle. For the GCM, two active electrodes were placed proximally so that one electrode was on each muscle. Specific recordings from the lateral aspect were obtained by placing active electrodes $2 \mathrm{~cm}$ apart, running parallel to the muscle fibers, just distal from the knee and $2 \mathrm{~cm}$ lateral to the midline [21]. The 


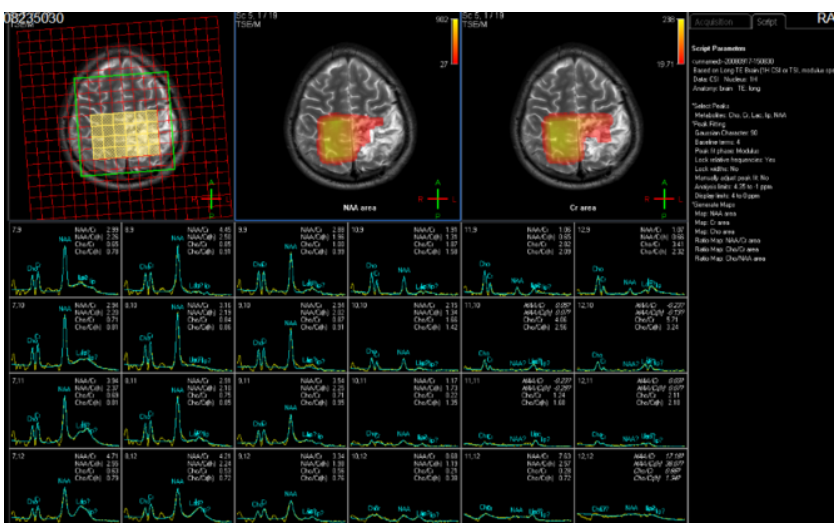

Fig. 1. Diagnostic brain MRI and spectroscopy.

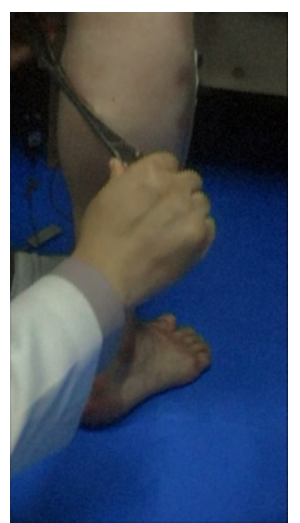

Fig. 2. Instrument-assisted neuromobilization (IASTM technique with Dr. You STM).

EMG signals were collected at sampling rate of $1024 \mathrm{~Hz}$ along with a 60- $\mathrm{Hz}$ notch filter; the bandpass filter was between 20 and $450 \mathrm{~Hz}$ and was analyzed using Telescan 3.06 software.

\section{Instrument-Assisted Neuromobilization (IASTM)}

The participant underwent Instrument-Assisted Neuromobilization of the GCM using a soft tissue mobilization (STM) instrument (Y1; Dr. You STM ${ }^{\circledR}$, Seed Tech Cooperation, Kyong-gi, Republic of Korea) (Figure 2). First, the participant was positioned prone with both feet hanging over the end of the exam table. The location of the shortness or tightness associated with hypertonicity of the GCM fiber was identified by the Y1 stroking method. Massage lotion was applied on the target location, and the Y1 tool was used to mobilize the short or tight muscle fiber in parallel using a stroking method in both non-weight-bearing (prone) and weight-bearing (standing) positions. The intervention was provided for 2 minutes in each position. Mobilization pressure was determined by the subject's tolerance to pressure and was kept comfortable to prevent pain-induced noxious stimulation or reflexive activation [22].

\section{Results}

Figure 1 presents diagnostic MRI and spectroscopy of the brain, which revealed an acute hemorrhage in the left middle cerebral artery territory, which involved moderate lesions along the corticospinal tract.

As illustrated in Figure 3, EMG amplitude analysis showed that prior to the intervention, the GCM was more significantly activated than the TA during ankle dorsiflexion, suggesting abnormal hypertonic GCM activity common in stroke. However, after the intervention, the GCM was deactivated by $43 \%$, and TA activation increased by $150 \%$, indicating IASTM-induced inhibition of the overactive GCM. 


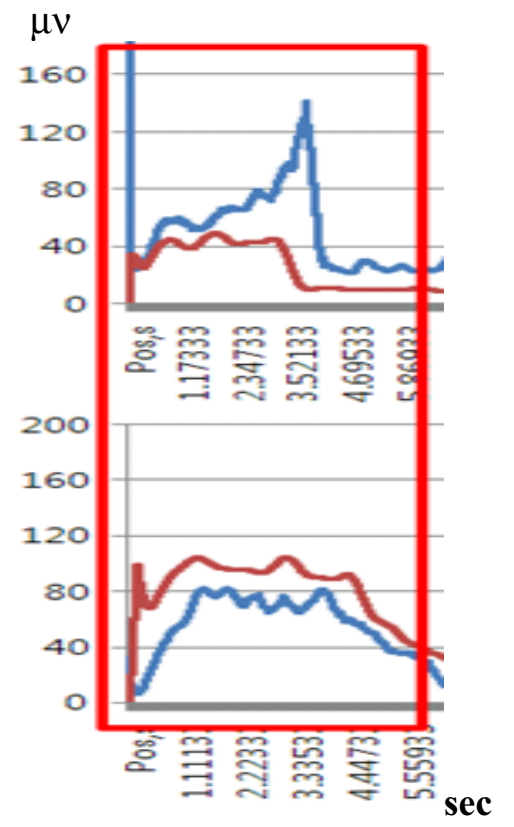

Fig. 3. Raw EMG activity of TA (red line) and GCM (blue line) before (upper) and after (bottom) intervention.

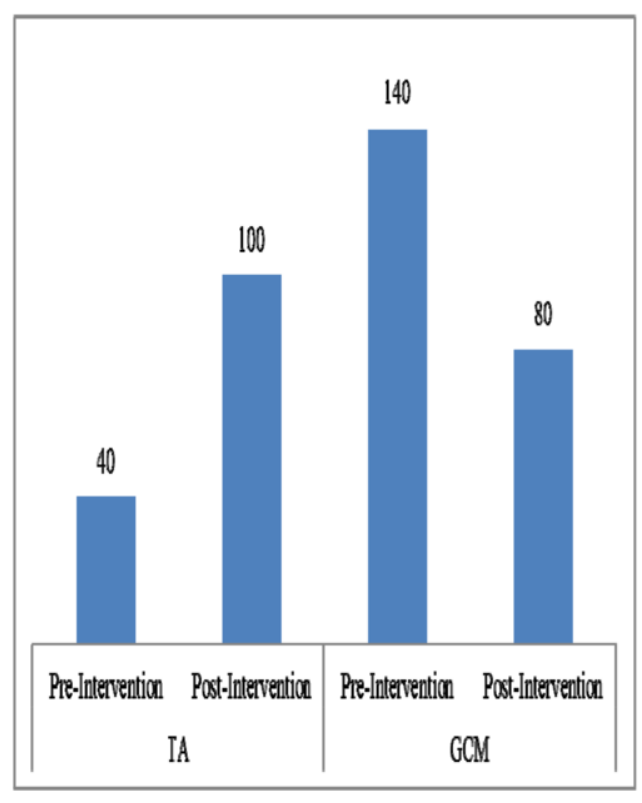

Fig. 4. EMG analysis of TA and GCM amplitudes $(\mu \nu)$ before and after intervention.

\section{Discussion}

The present study demonstrates compelling therapeutic effects for IASTM on GCM and TA muscle activation and the associated motor control mechanism. The IASTM technique was useful to decrease overactive GCM activity and reciprocally increase TA muscle activity in a stroke case. This finding has important clinical ramifications for stroke patients with gait impairment due to hypertonic or spastic plantarflexion $[23,24]$.

Our EMG analysis showed that before the intervention, the ankle plantarflexor (GCM) was overactive, even during ankle dorsiflexion movement when it should be relaxed or inhibited; the ankle dorsiflexor should be activated. Interestingly, after 2 minutes of the neuromobilization technique, abnormally hypertonic GCM activity was decreased by $43 \%$, and TA activation increased by $150 \%$, indicating IASTM-induced reciprocal inhibition effects of the overactive GCM (Figure 4). This finding supports Janda's notion of reciprocal inhibition of hypertonic activation of antagonist GCM and concurrent facilitation of the agonist TA activation [25]. To date, little or no evidence validates the effects of conventional manual neuromobilization techniques on hypertonic antagonist GCM affecting gait performance in stroke patients. Only a few studies $[19,26]$ have attempted to examine such techniques. It has been reported that IASTM was beneficial for inhibition of the hypertonic muscle and an increase in muscle fiber length in tight or shortened muscles in healthy subjects $[19,26]$. Recent EMG study investigated potential inhibition effects of IASTM on overactive and shortened hamstring muscles in 45 adults with hamstring shortness [20]. Significantly decreased hamstring muscle activation and improved quadriceps muscle activation were associated with terminal knee extension angle following the application of IASTM [20]. 
In this study, the IASTM neuromobilization technique showed a promising improvement of neuromuscular imbalance between TA and GCM activations in a stroke case. IASTM could be used as an alternative technique to alleviate the physical demands and associated common musculoskeletal impairments of among manual therapists when manual techniques with improper biomechanics are repetitively used over time [18].

Future studies should examine the long-term inhibition effects of IASTM in stroke patients with a larger sample size.

\section{References}

[1] G.M. Harper, The short-term effect of Graston Instrument-Assisted Soft Tissue Mobilization (GISTM) on supraspinatus tendinosis and it's concomitant findings. Master's Thesis, Durban Institute of Technology, 2006.

[2] S.J. Olney, Quantitative evaluation of co-contraction of knee and ankle muscles in normal walking, in: Biomechanics, Champaign, IL: Human Kinetics Publishers, 1985, pp. 431-435.

[3] R.R. Young, Physiologic and pharmacologic approaches to spasticity, Neural. Clin. 5 (1987), 529-539.

[4] K. Falconer and D.A. Winter, Quantitative assessment of co-contraction at the ankle joint in walking, Electromyogr. Clin. Neurophysiol. 25 (1985), 135-149

[5] G. Frost, J. Dowling, K. Dyson and O. Bar-Or, Cocontraction in three age groups of children during treadmill locomotion, J. Electromyogr. Kinesiol. 7 (1997), 179-186

[6] P. Page, C. Frank and R. Lardner, Structural and functional approaches to muscle imbalance, in: Assessment and treatment of muscle imbalance: The Janda approach, Human Kinetics Publishers, 2009, pp. 3-11.

[7] C.S. Sevier, O.A. Weisz, M. Davis and C.E. Machamer, Efficient export of the vesicular stomatitis virus G protein from the endoplasmic reticulum requires a signal in the cytoplasmic tail that includes both tyrosine-based and di-acidic motifs, Mol. Biol. Cell. 11 (2000), 13-22.

[8] N.H. Mayer, Clinicophysiologic concepts of spasticity and motor dysfunction in adults with an upper motor neuron lesion, Muscle Nerve. Suppl. 6 (1997), S1-13.

[9] R. Tanaka, Reciprocal Ia inhibition during voluntary movements in man, Exp. Brain Red. 21 (1974), 529-540

[10] N. Yanagisawa, R. Tanaka and Z. Ito, Reciprocal Ia inhibition in spastic hemiplegia of man, Brain 99 (1976), $555-574$.

[11] C. Crone, H. Hultborn, B. Jespersen and J. Nielsen, Reciprocal Ia inhibition between ankle flexors and extensors in man, J. Physiol. 389 (1987), 163-185.

[12] C. Crone and J. Nielsen, Spinal mechanism in man contributing to reciprocal inhibition during voluntary dorsiflexion of the foot, J. Physiol. 416 (1989), 255-272.

[13] C. Crone, Reciprocal inhibition in man, Dan. Med. Bull. 40 (1993), 571-581.

[14] A.F. Thilmann, S.J. Fellows and H.F. Ross, Biomechanics changes at the ankle joint after stroke, J. Neural. Neurosurgical Psychiatry 54 (1991), 134-139.

[15] J.B. Feland, J. Myrer and R. Merrill, Acute changes in hamstring flexibility: PNF versus static stretch in senior athletes, Phys. Ther. Sport. 2 (2001), 186-193.

[16] D.H. Kim, Effects of soft tissue mobilization techniques on neuromotor control and stiffness in hamstring shortness, Master's Thesis, Graduate School Yonsei University, 2014.

[17] M.J. Leathley, J.M. Gregson, A.P. Moore et al., Predicting spasticity after stroke in those surviving to 12 months, Clin. Rehabil. 18 (2004), 438-443

[18] J.E. Cromie, V.J. Robertson and M.O. Best, Work-related musculoskeletal disorders in physical therapists: prevalence, severity, risks, and responses, Phys. Ther. 80 (2000), 336-351.

[19] S. Fowler, J.K. Wilson and T.L. Sevier, Innovative approach for the treatment of cumulative trauma disorders, Work $\mathbf{1 5}$ (2000), 9-14.

[20] C. Lewis and T.W. Flynn, The use of strain-counterstrain in the treatment of patients with low back pain, J. Man. Mani. Ther. 9 (2001), 92-98.

[21] E. Criswell, Cram's introduction to surface electromyography, Jones \& Bartlett Publishers, 2010, pp. 371-374.

[22] D. Hopper, S. Deacon, S. Das, A. Jain, D. Riddell, T. Hall and K. Briffa, Dynamic soft tissue mobilization increases hamstring flexibility in healthy male subjects, Br. J. Sports Med. 39 (2005), 594-598.

[23] A. Lamontagne, F. Malouin and C.L. Richards, Locomotor-specific measure of spasticity of plantarflexor muscles after stroke, Arch. Phys. Med. Rehabil. 82 (2001), 1696-1704. 
[24] F. Malouin, C. Bonneau, L. Pichard and D. Corriveau, Non-reflex mediated changes in plantarflexor muscles early after stroke, Acta. Phys. Med. Rehabil. 29 (1997), 147-153.

[25] T. Sinkjaer and I. Magnussen, Passive, intrinsic, and reflex-mediated stiffness in the ankle extensors of hemiplegic patients, Brain 117 (1994), 355-363.

[26] C.S. Sherrington, On reciprocal innervation of antagonistic muscles, Proc. R. Soc. Lond. B. Biol. Sci. 79B (1907), 337349. 